\title{
MANUTENÇÃO PREDITIVA APLICADA A AMBIENTES DE MISSÃO CRÍTICA DE SUPERCOMPUTAÇÃO UTILIZANDO INTELIGÊNCIA ARTIFICIAL: UMA REVISÃO SISTEMÁTICA DE LITERATURA
}

\author{
André Luis da Cunha Dantas Lima1; Vítor Moraes Aranha²; Erick Giovani Sperandio \\ Nascimento ${ }^{2}$
}

1 SENAI CIMATEC; Salvador/BA; andrelcdI@gmail.com

2 SENAI CIMATEC; Salvador/BA

Resumo: Os ambientes de missão crítica de supercomputação (AMCS) estão diretamente relacionados a equipamentos energizados ininterruptamente, em que qualquer falha prejudica na sua disponibilidade e no aumento dos custos operacionais. Conhecer os estudos sobre esta problemática permite verificar soluções existentes que detectem automaticamente padrões de comportamento e falhas futuras. Assim, esta revisão sistemática da literatura tem como objetivo identificar e sumarizar as contribuições neste ramo específico do conhecimento, visando uma maior qualidade e confiabilidade dos resultados, os quais foram classificados e sintetizados. Este estudo contribui tanto para a área de manutenção preditiva aplicada em AMCS quanto para área de ciência da computação com aplicação da inteligência artificial.

Palavras-Chave: Manutenção Preditiva; Supercomputação; Computação de alto desempenho; Inteligência artificial; aprendizado profundo.

\section{PREDICTIVE MAINTENANCE APPLIED TO SUPERCOMPUTING CRITICAL ENVIRONMENTS USING ARTIFICIAL INTELLIGENCE: A SYSTEMATIC LITERATURE REVIEW}

\begin{abstract}
Mission-critical supercomputing environments are closely related to constantly powered devices, where faults and failures directly affect availability and operational costs. Learning from previous investigations on this matter makes it possible to verify existing solutions that detect patterns of behavior and predict equipment failures. Therefore, this systematic review aims to identify and summarize the contributions to this field, aiming to obtain more quality and reliability from the achieved results by a carefully designed classification. This study contributes both to the field of predictive maintenance applied to mission-critical environments and to the field of computer science, with artificial intelligence.
\end{abstract}

Keywords: Predictive maintenance; High performance computing; HPC; Artificial Intelligence; deep learning; 


\section{INTRODUÇÃO}

Com o crescimento de serviços na nuvem, boa parte do processamento de dados antes feito em desktops ou servidores locais agora é feito por grandes datacenters com milhares de computadores interconectados. O aumento da demanda na alocação de nós computacionais em ambientes de HPC (High-performance computing) é progressivo principalmente para finalidades de simulação e testes de modelos computacionais, os quais necessitam de alto poder computacional para obtenção de resultados mais rápidos e que deem suporte mais preciso às indústrias/empresas para tomadas de decisões estratégicas e/ou de mercado.

Para estes tipos de ambientes, é imprescindível prover uma infraestrutura redundante ativa a ser acionada em incidentes ou situações emergenciais em casos de falha ou falta destes equipamentos. Como consequência imediata, gera-se um aumento no consumo energético e custo de operação, principalmente quando envolvem ambientes de missão crítica que estão diretamente relacionados a soluções redundantes de equipamentos energizados 24 horas por dia, 7 dias da semana.

Dessa forma, a manutenção preditiva se torna um método muito interessante a ser aplicado em ambientes de missão crítica com a principal finalidade de predizer as condições reais de funcionamento dos equipamentos com base em dados históricos que informam o seu desgaste ou processo de degradação, objetivando encontrar defeitos em estágio inicial, quando estes ainda são falhas potenciais, o que possibilitaria saná-los antes do agravamento e evolução para uma falha funcional do equipamento.

Entretanto, devido a quantidade de dados e logs (registros) gerados por minuto num ambiente de computação de alto desempenho, através do supercomputador e dos diversos equipamentos utilitários e acessórios espalhados neste ambiente, tornase impossível ao técnico-operador identificar, ordenar, categorizar, combinar e analisar os dados de forma isolada a fim de compreender e produzir um significado da informação. Muitas vezes, um dado coletado pode ser crítico para o funcionamento do conjunto, mas neste cenário é simplesmente descartado ou desviado sem ter a devida importância desde a sua fonte geradora até o receptor, prejudicando a correta análise pela equipe de monitoramento para o devido controle de manutenção.

Diante deste contexto, a manutenção preditiva aliada a técnicas de Inteligência Artificial (IA) pode ser uma solução, já que a integração e o uso de dados históricos acumulados e dados operacionais em tempo real permitem que algoritmos/modelos analíticos voltados para a manipulação de grandes volumes de dados (Big Data) detectem antecipadamente padrões e determinem o comportamento futuro do equipamento (ativo monitorado), com uma acurácia cada vez maior. À medida que este sistema é capaz de aprender tais padrões e prever falhas, ele poderá oferecer suporte à tomada de decisão para a realização de paradas planejadas, diminuindo assim, os tempos de desligamento, os custos de manutenção e as corretivas, por consequência, aumenta a disponibilidade do equipamento, os níveis de produtividade, confiabilidade e desempenho de todo o ambiente.

Para fundamentar este estudo é necessário identificar as contribuições científicas sobre esta linha de pesquisa e sumarizar as evidências existentes, visando uma maior confiabilidade e qualidade de resultados. Por isso, a elaboração de uma revisão sistemática como principal método de síntese, viabiliza e apoia a integração 
das informações de um conjunto de estudos realizados separadamente que podem apresentar resultados conflitantes e/ou coincidentes, bem como auxiliar na orientação para investigações futuras sobre o tema relacionado.

\section{MANUTENÇÃO PREDITIVA}

Segundo [1], existem quatro métodos de manutenção industrial: corretiva (conserta quando quebra), preventiva (programa atividades para manutenção), proativa (elimina os defeitos para melhorar a performance) e preditiva. A manutenção preditiva é a solução mais eficiente e promissora em relação às demais, pois é um método prático que possibilita avaliar a condição do equipamento através da coleta de dados/registros de eventos anteriores com o intuito de predizer o tempo em que a falha do equipamento estiver próxima de acontecer, possibilitando uma ação antecipada de manutenção do equipamento evitando quebras e paradas não planejadas. Este método reduz a incerteza, o tempo de parada, gerencia riscos e possibilita a redução dos custos, sendo possível utilizá-lo em muitos cenários da Indústria.

\section{INTELIGÊNCIA ARTIFICIAL (IA)}

O objetivo da IA está voltado para a realização de tarefas cognitivas, as quais os humanos são atualmente melhores, utilizando paradigmas/algoritmos desenvolvidos que necessitam de máquinas para aprende-las. Um sistema de IA possui a capacidade de representar, raciocinar e aprender através da experiência [2]. O aprendizado profundo (Deep Learning) é um subcampo específico do aprendizado de máquina (Machine Learning) que trata camadas de aprendizado de forma sucessiva utilizando dados de representações cada vez mais significativos. Dessa forma, para tratamento e correlação de grande volume de dados coletados, o uso desta técnica possibilitará aprender conceitos complexos a partir de conceitos mais simples utilizando várias etapas, ou seja, o operador humano especifica formalmente todo o conhecimento necessário que a máquina necessita, armazenando assim uma hierarquia de conceitos [3].

\section{METODOLOGIA}

Este estudo foi realizado como uma revisão sistemática da literatura, com base nas diretrizes originais citadas por [4]. Neste caso, os objetivos da revisão são: identificar algoritmos de inteligência artificial para predição de falhas aplicável em ambiente de missão crítica de supercomputação; identificar metodologias de pesquisa usadas em contextos equivalentes e seus resultados, além de mapear quais os requisitos essenciais para melhor definir a técnica de aprendizado de máquina para predição de séries temporais multivariadas aplicadas a equipamentos computacionais. As etapas do método de revisão sistemática da literatura estão documentadas abaixo.

\subsection{Processo de Pesquisa}


Primeiramente foram definidas as seguintes questões norteadoras para 0 levantamento das publicações relevantes:

Q1. Existem estudos sobre manutenção preditiva aplicada a ambientes de missão crítica de supercomputação utilizando inteligência artificial?

Q2. Existem algoritmos de inteligência artificial para predição de falhas aplicável em equipamentos computacionais?

Q3. Quais requisitos essenciais para melhor definir a técnica de correlação dos dados coletados?

Q4. Quais requisitos essenciais para melhor definir a técnica de aprendizado de máquina para predição de séries temporais multivariadas aplicadas a equipamentos computacionais?

Após essa definição, iniciou-se o processo de busca automática por meio da criação de filtros nas bases de dados da IEEE Xplore Digital Library, Science Direct e SpringerLink, utilizando as diversas combinações dos descritores ou palavras-chaves: "high performance computing", "predictive maintenance", "artificial intelligence", "deep learning", "computação de alto desempenho", "manutenção preditiva", "inteligência artificial", "aprendizado profundo".

\subsection{Critérios de Inclusão e Exclusão}

Foram definidos como critérios para inclusão dos estudos a revisão por pares, artigos originais em periódicos ou conferências entre 2010 e 2019 e a redação em um dos idiomas Inglês ou Português. Publicações que não se enquadravam nessas categorias, sem enfoque em ambientes industriais, HPC, cluster ou datacenter, assim como, resenhas, bibliografias, editoriais e relatórios, não foram considerados nesta pesquisa.

A avaliação da qualidade para inclusão dos trabalhos também foi baseada as repostas as seguintes questões:

Q1. Foi especificado e testado o algoritmo de IA proposto para correlação dos dados coletados no estudo em ambiente de computação de alto desempenho, clusters ou datacenter?

Q2. Foi apresentado a metodologia/método de avaliação do estudo?

Q3: É apresentada a forma e frequência da coleta dos dados? Estão na mesma base temporal?

Q4. Os dados coletados são rotulados com eventos de alarme/falha presentes? Existe informação sobre a transição do modo normal e falha?

Q5: As conclusões do estudo deixam claro se o estudo proposto obteve sucesso ou não?

Cada questão foi respondida com Sim (S), Parcial (P) ou Não (N) com as notas $1,0,5$ ou 0 pontos, respectivamente. Dessa forma, quanto mais próximo da nota 5,0 , maiores as chances do artigo ser relevante ao tema desta pesquisa e, por conseguinte, estar inserido no contexto das questões que nortearam esta pesquisa.

\section{RESULTADOS E DISCUSSÃO}


Na primeira etapa da seleção de artigos, no total, foram encontrados 964. A Tabela 1 apresenta o quantitativo dos estudos encontrados durante cada etapa de busca nas bases selecionadas.

Tabela 1. Total de estudos encontrados por etapa da revisão sistemática.

\begin{tabular}{|l|c|c|c|}
\hline \multicolumn{1}{|c|}{ BASE } & ETAPA 1 & ETAPA 2 & ETAPA 3 \\
\hline IEEE Xplore Digital Library & 30 & 11 & 6 \\
\hline SpringerLink & 756 & 24 & 2 \\
\hline ScienceDirect & 178 & 12 & 3 \\
\hline
\end{tabular}

A etapa 1 correspondeu à aplicação dos critérios de inclusão e exclusão definidos anteriormente. Em seguida, na etapa 2, buscou-se identificar os termos de pesquisa no título e no resumo/abstract, resultando em 47 estudos. Na etapa 3, foram considerados apenas os estudos em que a introdução e a conclusão são voltados para o tema deste trabalho, resultando em 11 estudos apresentados na Tabela 2. A partir desta seleção, os estudos foram lidos na íntegra aplicando a Avaliação de Qualidade definida na Seção 4.2 para aqueles que apresentaram notas acima de 4,0. A Tabela 3 apresenta as respostas para cada pergunta desta avaliação e o somatório da pontuação na última coluna.

Tabela 2. Artigos selecionados na Etapa 3.

\begin{tabular}{|c|c|c|}
\hline ARTIGO & ANO & TÍTULO \\
\hline A1 & 2017 & $\begin{array}{l}\text { A Hybrid Deep Representation Learning Model for Time Series } \\
\text { Classification and Prediction }\end{array}$ \\
\hline A2 & 2012 & $\begin{array}{l}\text { A two-stage equipment predictive maintenance framework for } \\
\text { high-performance manufacturing systems }\end{array}$ \\
\hline A3 & 2015 & Cloud-based framework for advanced maintenance tasks \\
\hline A4 & 2018 & Failure Prediction Model for Predictive Maintenance \\
\hline A5 & 2015 & $\begin{array}{l}\text { Machine Learning for Predictive Maintenance: a Multiple } \\
\text { Classifier Approach }\end{array}$ \\
\hline A6 & 2013 & $\begin{array}{l}\text { Machinery Condition Prediction Based on Wavelet and Support } \\
\text { Vector Machine }\end{array}$ \\
\hline A7 & 2012 & $\begin{array}{l}\text { Autonomous and adaptive procedure for cumulative failure } \\
\text { prediction }\end{array}$ \\
\hline A8 & 2018 & $\begin{array}{l}\text { Curve-Registration-Based Feature Extraction for Predictive } \\
\text { Maintenance of Industrial Equipment }\end{array}$ \\
\hline A9 & 2018 & $\begin{array}{l}\text { A big data driven sustainable manufacturing framework for } \\
\text { condition-based maintenance prediction }\end{array}$ \\
\hline A10 & 2018 & $\begin{array}{l}\text { A new subset based deep feature learning method for intelligent } \\
\text { fault diagnosis of bearing }\end{array}$ \\
\hline A11 & 2019 & $\begin{array}{l}\text { Forecasting remaining useful life: Interpretable deep learning } \\
\text { approach via variational Bayesian inferences }\end{array}$ \\
\hline
\end{tabular}

Tabela 3. Avaliação de Qualidade.

\begin{tabular}{|c|c|c|c|c|c|c|}
\hline ARTIGO & Q1 & Q2 & Q3 & Q4 & Q5 & PONTOS \\
\hline A1 & P & S & S & N & S & 3,5 \\
\hline A2 & P & S & S & P & S & 4,0 \\
\hline A3 & N & S & P & N & S & 2,5 \\
\hline A4 & P & S & S & P & S & 4,0 \\
\hline
\end{tabular}




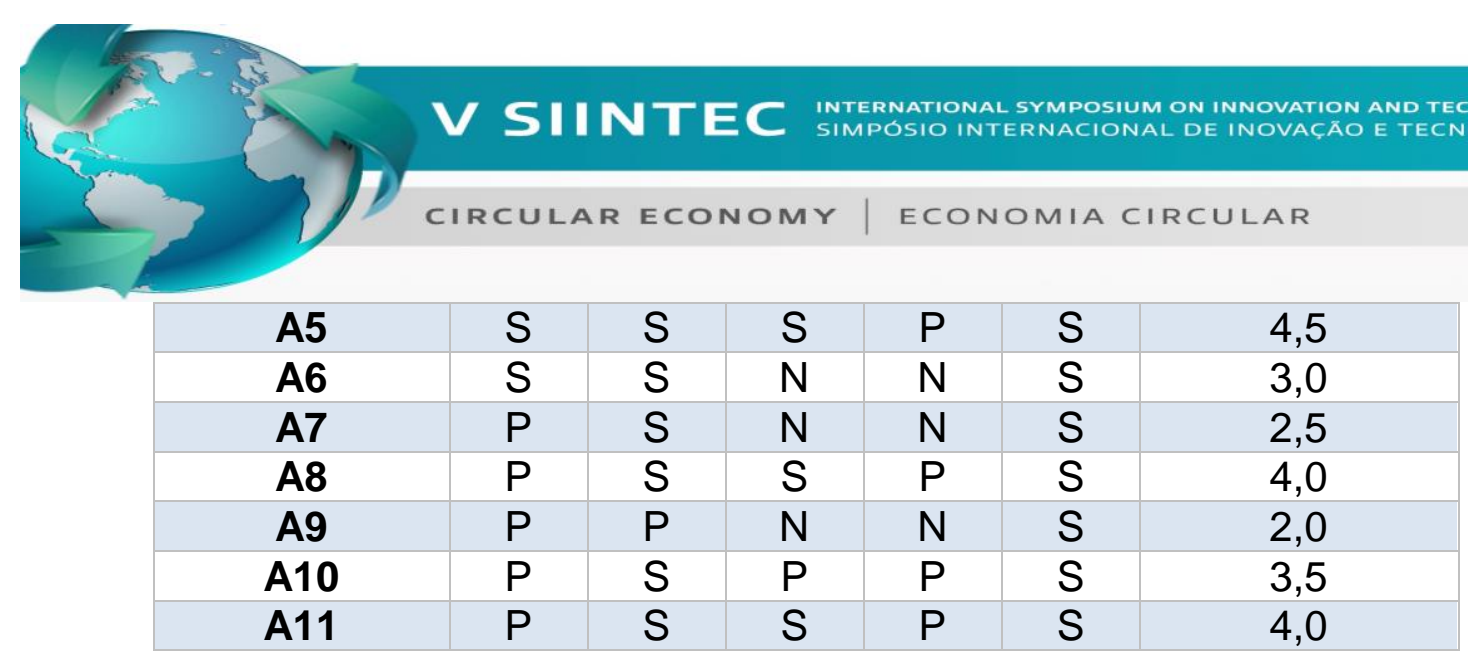

Após a aplicação da Avaliação de Qualidade, restaram 05 estudos com notas iguais ou acima de 4,0. Observa-se que os trabalhos desenvolvidos vêm se aproximando do tema para aplicação de técnicas de Inteligência Artificial em ambientes que necessitam predizer as falhas/faltas em equipamentos críticos envolvidos em soluções industriais, os quais não podem parar.

No estudo apresentado no $\mathrm{A} 2$, um método em dois estágios para predição de falhas foi experimentado em uma planta de fabricação de semicondutores. A forma mais comum de detectar possíveis falhas em equipamentos é através da coleta de dados de sensores e logs. Partindo disso, relatórios históricos dos equipamentos foram coletados em campo e treinados em uma rede neural com um algoritmo de propagação-reversa (backpropagation). Em um segundo passo, um algoritmo genético foi usado para circunstâncias em que a propagação-reversa se demonstrou incapaz de escapar de zona de pontos mínimos locais. Verificou-se que a técnica utilizada com abordagem evolutiva alcançou acurácia apenas de $3 \%$ inferior às técnicas de propagação reversa tradicionais, enquanto apresentou melhor desempenho na busca e nos cálculos das zonas de mínimos globais [5].

A técnica apresentada em A4, aplica um modelo de aprendizagem de máquina em arquivos de logs de máquinas de ATM (Automated Teller Machines) para predição de falhas. O processo de preparação dos dados considera os relatórios de erros mais recorrentes e descarta aqueles com menor frequência para estabelecer uma melhor correlação entre as principais causas ou gatilhos para ocorrência de falhas. $O$ desempenho do modelo é medido através da relação entre accuracy e recall e a probabilidade de falha em um dispositivo é calculada como um valor entre 0 e 1 . Os autores discutiram a generalização da técnica e a ampliação dos features o que possibilita o avanço na pesquisa para a replicação em áreas saúde, indústria, entre outras [6].

Um método de manutenção preditiva através de abordagem de classificadores múltiplos foi adotado em A5 e testada em um benchmark de fábrica de semicondutores. A técnica foi capaz de lidar com dados de várias dimensões através do treinamento de módulos de classificação com diferentes horizontes de predição. $O$ algoritmo foi testado com duas técnicas amplamente utilizadas na literatura, Support Vector Machines (SVM) e k-Nearest Neighbors (kNN), os quais geraram benefícios no custo de operação quando comparados a abordagens que aplicam somente manutenção preventiva. No melhor caso, obteve-se a acurácia de 98,52\% utilizando SVM e $98,51 \%$ utilizando com kNN. Mesmo assim, os autores observaram que há possibilidade de melhoria nos resultados com a investigação do uso de Relevance Vector Machines com o objetivo de tratar a alta dimensionalidade dos tipos de dados comuns à manutenção preditiva [7].

Nos estudos apresentados por [8] e [9], o uso de modelos Low Short Term Memory (LSTM) é utilizado para aprendizado de séries temporais devido a capacidade 
desta técnica "lembrar" de valores anteriores melhorando assim a eficácia de suas predições.

A pesquisa desenvolvida no A11 propôs uma solução de compromisso entre interpretatividade do modelo de aprendizado de máquina e predição da vida útil de componentes através de inferências Bayesianas variacionais. A técnica foi testada utilizando o dataset Turbofan, de motores de aeronaves. Os resultados alcançaram níveis superiores comparados às abordagens tradicionais de modelos de machine learning, pois quando foram aplicadas as inferências Bayesianas a uma rede LSTM percebeu-se uma melhora de $37,12 \%$ no erro em relação a técnica de random forest. Este método também obteve grau razoável de interpretatividade permitindo a interpretação e inferência do conteúdo do aprendizado da rede. Após os autores realizarem testes empíricos no modelo criado, observou-se que o uso de recentes investigações que reformulam dropout como uma aproximação Bayesiana não ofereceram ganhos, o que abre margem para futuras investigações sobre como se beneficiar desta técnica de regularização [8].

Para melhorar a precisão e o desempenho do modelo de predição, o estudo A8 propôs uma técnica de extração de features para correlação time-lagged. Essa abordagem se mostrou capaz de reduzir os tempos de treino da rede enquanto preservava a precisão das predições. O modelo foi testado em três métodos de machine learning (rule-based, d-LSTM e dp-LSTM), entretanto os melhores resultados foram obtidos através do uso da rede LTSM, atingindo um recall médio de $85,6 \%$ e precisão de 78\% [9].

A manutenção preditiva permite minimizar os custos de manutenção de equipamentos, maximiza o tempo de operação e preserva sua integridade ao reduzir os riscos de falhas, possibilitando assim, uma antecipação de medidas preventivas para evitar a quebra ou falta do equipamento/ativo.

Considerando o sucesso de modelos de aprendizagem de máquina para predição de eventos em séries temporais e o contínuo monitoramento de equipamentos de missão crítica, o uso de redes neurais e aprendizado profundo se mostram um caminho viável para este domínio de aplicação [10].

Relatórios de erros e dados sensoriais coletados por equipes de campo ou por ferramentas de instrumentação são frequentemente suficientes para obter previsões de comportamento de equipamentos de missão crítica. Conforme [6], em alguns casos é possível desconsiderar eventos de aviso ou falha menos frequentes em favor daqueles mais comuns, uma vez que isso pode significar uma tendência merecedora de atenção por parte dos times de operação/monitoramento.

Finalmente, observa-se que os autores dos artigos selecionados buscaram desenvolver pesquisas na área de manutenção preditiva utilizando métodos de machine learning, em especial as redes LSTM devido sua eficácia de predições em series temporais. Porém, o domínio das investigações foi focado em ambientes industriais.

\section{CONCLUSÃO}

O resultado desta revisão sistemática proporcionou uma visão do estado da arte nas pesquisas ora desenvolvidas, no período de 2010 a 2019, direcionadas para manutenções preditivas e que utilizaram técnicas de machine learning já 
estabelecidas na literatura, como por exemplo LSTM. Por outro lado, verificou-se que há uma oportunidade de futuras investigações para aplicação de métodos e técnicas de deep learning em ambientes de supercomputação de missão crítica para fins de manutenção preditiva, pois a pesquisa aqui realizada demonstrou que não existe nenhum estudo previamente feito em que se aplicaram esses conceitos para este tipo de aplicação, de sorte que o grande volume de dados coletados envolvendo vários equipamentos possibilitará um maior entendimento dos comportamentos dos ativos/dispositivos de forma preditiva e a melhoria do desempenho das previsões de falhas neste ambiente utilizando Inteligência Artificial, o que poderá contribuir para redução de custos operacionais.

\section{Agradecimentos}

Agradecemos ao Centro de Supercomputação para Inovação Industrial do SENAI CIMATEC e a BULL por proporcionar a infraestrutura e o ambiente de trabalho para execução desta pesquisa.

\section{REFERÊNCIAS}

${ }^{1}$ YE, Chen. A System Approach to Implementation of Predictive Maintenance with Machine Learning. Massachusetts Institute Of Technology, 2018. Disponível em: $<$ https://www.researchgate.net/publication/328333169_A_system_approach_to_implementati on_of_predictive_maintenance_with_machine_learning >. Acesso em: 28 jul. 2019.

2 HAYKIN, Simon. Redes neurais: princípios e prática. ed. Bookman Editora, 2007.

${ }^{3}$ GOODFELLOW, I.; BENGIO, Y.; COURVILLE, A. Deep Learning. MIT Press, 2016.

${ }^{4}$ KITCHENHAM, Barbara; PEARL BRERETON, O.; BUDGEN, David; et al. Systematic literature reviews in software engineering - A systematic literature review. Information and Software Technology, v. 51, n. 1, p.7-15, 2009. DOI 10.1016/j.infsof.2008.09.009. Disponível <https://www.sciencedirect.com/science/article/abs/pii/S0950584908001390>. Acesso em: 27 jul. 2019.

${ }^{5}$ BIN HU; PANG, C. K.; LUO, M.; et al. A two-stage equipment predictive maintenance framework for high-performance manufacturing systems. In: 2012 7th IEEE Conference on Industrial Electronics and Applications (ICIEA), p. 1343-1348, 2012.

${ }^{6}$ SUSTO, G. A.; SCHIRRU, A.; PAMPURI, S.; et al. Machine Learning for Predictive Maintenance: A Multiple Classifier Approach. IEEE Transactions on Industrial Informatics, v. 11, n. 3, p. 812-820, 2015.

${ }^{7}$ MISHRA, K.; MANJHI, S. K. Failure Prediction Model for Predictive Maintenance. In: 2018 IEEE International Conference on Cloud Computing in Emerging Markets (CCEM), p. 7275, 2018.

${ }^{8}$ KRAUS, Mathias; FEUERRIEGEL, Stefan. Forecasting remaining useful life: Interpretable deep learning approach via variational Bayesian inferences. Decision Support Systems, p. 113-100, 2019.

9 ZHANG, Shouli; LI, Xiaohong; WANG, Jianwu; et al. Curve-Registration-Based Feature Extraction for Predictive Maintenance of Industrial Equipment. In: ROMDHANI, Imed; SHU, Lei; TAKAHIRO, Hara; et al (Orgs.). Collaborative Computing: Networking, Applications and Worksharing. Springer International Publishing, p. 253-263, 2018.

10 Zhang, K., Xu, J., Min, M.R., et al.: Automated IT system failure prediction: a deep learningapproach. In: 2016 IEEE International Conference on Big Data, pp. 1291-1300, 2016. 